\title{
Aspect Design with the Building Block Method
}

\author{
Jürgen K. Müller \\ Philips Research Laboratories, Prof. Holstlaan 4 (WLp), NL - 5656 AA Eindhoven \\ mueller@natlab.research.com
}

Key words: Software architecture, software component, object-oriented modelling, function-oriented modelling, design method

\begin{abstract}
Aspects are a way to supplement object-oriented modelling with functionoriented modelling. The Building Block Method (BBM) identifies 3 dimensions of independent design. Besides an object dimension and a process dimension also an aspect dimension is present. Object design, process design and function design are done independently. Building Blocks (BB), which are software components, cluster functionality according to criteria such as configurability in a product family and incremental system integration. The BBM is used to design families of telecommunication infrastructure, digital broadcasting and medical imaging systems. The paper introduces the concept of aspects and shows how they are identified and used in the BBM.
\end{abstract}

\section{INTRODUCTION}

Today's electronic systems implement more and more of their functionality in software. The flexibility of software and the price erosion of standard computing hardware further this trend. Despite all kinds of modularity in hardware and software, the integrating system characteristics of the larger systems are always implemented in software. These architectural software structures are much harder to change than more local hardware and software parts. The continuous evolutionary development requires that software and hardware needs to be changed and extended in a piecemeal way. To enable this the basic architectural structures must be designed to do this with moderate effort.

The original version of this chapter was revised: The copyright line was incorrect. This has been corrected. The Erratum to this chapter is available at DOI: 10.1007/978-0-387-35563-4_35 
The use of object-oriented modelling has advanced the conceptual level of implementations as consisting of a network of objects. For small and medium size systems this may be enough. Large systems, however, easily become monolithic, i.e., an unmanageable web of objects. They therefore need more modularity and more locality of changes in an evolutionary development situation. Object-oriented concepts should be complemented by other concepts from other modelling techniques, for example functional modelling. The goal is to create systems that are modelled naturally for their application domain [10]. BB aspect design is a means to cope with this problem.

The next section gives an introduction to the BBM. Section 3 defines software aspects and shows by means of examples how software aspects are derived from a system level perspective. Software aspects are an important means to relate software functionality to the overall system functionality. Section 4 and 5 look at the consequences for components and componentbased development. Section 6 compares the aspects in the BBM with other approaches.

\section{OVERVIEW OF THE BBM}

The BBM is a component based [18] design method for the development of the software for central controllers of embedded systems. These central controllers integrate, control, and manage the overall system. They are points of great complexity.

Many of the concepts that we will present can (or better, should) be used for all of the software of an embedded system; in fact they are useful in the structuring of any large software system. However, we focus on the specific problem of central controllers to be able to reason very specifically, which would be more difficult if we dealt with software systems in general [9]. Furthermore, the basic ingredients of the method presented have been tried out successfully in the design of these central controllers.

Experiences with the method stem from the development of systems in the telecommunications, video broadcasting and medical imaging domain. The most complete overview of the BBM can be found in [12]. Some quantitative figures are given in [16].

The method presented is designed to support the creation of product family architectures [4]. Composing a product from pre-manufactured components is how software reuse in a product family architecture is achieved [13]. The product management requirements of short lead time, low development effort, and high quality products have been translated into the architectural requirements of conceptual integrity, managed complexity, 
delta development, extensibility, reusability, configurability, and testability [12]. These requirements have been taken as the main ones to be achieved in a system architecture constructed via our method.

However, it is clear that architecture can only lay a good foundation on which the system is built. A good product needs more than just a good architecture. Errors can be made at all levels of system development: requirements, architecture, detailed design, implementation, deployment, documentation, to name the most important ones. In the next section we take a look at the architectural meta-model (AM-M) of the BBM.

\subsection{The AM-M of the building block method}

Traditionally the description of an architecture and its models have been very much dependent on the persons presenting them. Depending on the major problems to be solved by the architecture, or the personal preferences of the persons involved, structures like hardware boundaries, modules, processes or state machines have been presented as the architecture.

However, experiences from different projects show that besides the specific nature of a product the architecture had to address a number of common problems. These problems are, for example, the units in which a software system is broken down, composed and evolved; or the way in which processing resources are to be used by the application. The solutions to those problems are part of an architecture.

Different methods define different things to be part of an architecture. We call those parts of an architecture that are required by a method the architectural meta-model of a method.

The architectural meta-model of the BBM consists of

- a logical model,

- the building block design dimensions (BBDD), and

- components, the so-called building blocks (BB).

We explain the AM-M of the BBM in the rest of this section.

\subsubsection{Logical model}

The logical model is the model of the functionality of the systems to be built. It describes the externally observable (and the made-known internal) functionality. The language used is the one of the customer (or user) and of the product managers. The intention of the model is to precisely describe

- the "what" of the system.

- the environment of the system, e.g., to which interfaces it has to comply.

- other conditions for the system.

The "how" of the implementation is left to design. 


\subsubsection{Modelling language and domain modelling}

A lot of different modelling languages have been proposed. Examples include data flow diagrams, entity-relationship diagrams, state machines, object-oriented modelling. However, no standard has yet evolved, and it is questionable if that will ever happen.

If you look at a very mature domain, say cars, a lot of domain-specific objects are used to describe and compare cars. Also new cars are described in terms of the attributes of domain objects, e.g., the number of cylinders of the motor and its horse power, the type of gearbox, the interior design, its maximum speed, etc. In a new domain a description of a product uses many more functions of that product than attributes of domain objects. The objects are still a matter of design.

However, in many domains object-oriented modelling will be the modelling language of choice. The most prominent one is the Unified Modelling Language UML [6]. There are many methods to develop such an OO model.

As a domain matures and companies want to cover a complete application domain with their products the focus of the logical model changes. Being initially the model of the functionality of a product, the logical model is used to describe a complete application domain (together with a selection list for each specific product).

The BBM does not use a specific modelling language nor a specific method to develop such a logical model. It assumes that one exists and takes it as basis for the further architectural design.

\subsubsection{Design dimensions}

The idea of dimensional structuring is introduced to support intellectual control of system functionality. If common concepts or structures that apply to the same item can be separated such that there are no mutual restrictions, the concepts or structures are orthogonal; we can talk about design dimensions. Thus, design can be carried out independently for every dimension. Each item in the design space can be reduced (projected) to one specific dimension. The BBM identifies 3 design dimensions that will be motivated below. Of course functionality of the 3 dimensions has to be related. Design guidelines for each dimension and for relating the 3 are given. However, the BBM views every such relation as a design decision.

The first point is to separate object structuring from the use of execution units. Objects stem from the modelling of domain functionality. Execution units determine the use of processing resources for independent, cooperating and/or sequential actions. The designer should be free to do the process design without consequences for the object design. They build two 
orthogonal dimensions, i.e., a method of an object may be driven by one or more processes and a process may drive methods from different objects.

The second point is to introduce functional structuring in addition to object-based structuring. Those global functions that deal with potentially all objects (i.e., they cross-cut objects) are handled as aspects. Thus each object method in the system is part of an object and part of an aspect.

The BBM combines these two points, which leads to three design dimensions: the object dimension, the aspect dimension, and the process dimension (figure 1).

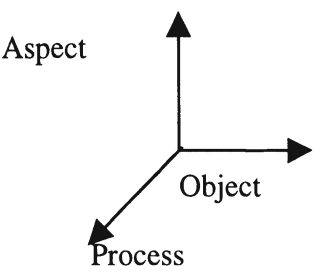

Figure 1. Design dimensions

In the BBM, an object method is defined to be the minimal block of functionality. This corresponds to a point in the design space. The first dimension is the object dimension, which covers the decomposition of the system into data units with their access procedures. The second dimension is according to aspects. Each aspect deals with a specific view of the functionality of the systems, such as recovery, configuration management, fault handling, etc. The whole system functionality is partitioned by the set of aspects. The third dimension is the process dimension, viz. the whole system is driven by a set of processes. The guidelines to identify and define objects and processes are not the subject of this paper.

The consequence is that design for each dimension can be made independent of the other two, which gives the freedom to make a design that is best suited for the application. The structuring in the design dimensions is a structuring at the meta-level of the system.

\subsubsection{System evolution and design dimensions}

With respect to system evolution, the aspect dimension is not handled in the same way as the object dimension. We assume that a system is primarily modelled using objects. For most application areas this gives the most stable modelling. The most common extensions of the system are via extended objects and new objects (i.e., there exists a locality of change).

The two design dimensions, object and aspect, are a specific union of object-oriented and functional modelling. Aspects are seen as a secondary 
form of modelling. They are functions that cross-cut all or most objects. To achieve stable software structures aspects should be standardised for a complete product family. Adding a new aspect affects all related objects.

Process design starts by looking for independence of objects. But for most systems we assume that a situation is aimed for where the structure of independent execution units is standardised, either in specific classes or in some rules that guide the creation of classes and instances (see Gomaa [7]). Without such rules the understanding of a large evolving system is very difficult.

Because of the above-described situation, that aspects and processes should be stable, we say that the evolution of the system is mainly in the object dimension.

\subsubsection{Components}

Components are deployment units that are identified in the architectural phase [18]. This means that they are present during all of the development phases. They are called Building Blocks (BB). They are the main focus of the BBM. An architecture identifies the BBs. A specific product is built out of BBs. This means that a BB has a specific representation in all the phases.

There are two important points for BBs: what is the content of a BB and what are the relations between BBs? Through the design dimensions the functionality of a complete system is designed. A BB usually consists a collection of objects. It does usually not contain complete processes or aspects. The set of $\mathrm{BBs}$ covers the complete functionality in a nonoverlapping way. There are certain criteria for identifying BBs; the main one is configurability. These criteria may also influence the design along the 3 design dimensions. A design process evolves typically in several steps until stability is reached.

A system architecture has to define inter- and intra-BB structures. Aspects are used for intra-BB structuring (see below). Inter-BB structures are defined via the concepts of layers, subsystems, classification of BBs, and a skeleton.

The include relations of all BBs form a partial order. Each BB resides in a layer [3] and can only use BBs in strictly lower layers. During the initialisation phase a $\mathrm{BB}$ in a higher layer binds itself to a BB in a lower layer (post-load linking). Through this binding $\mathrm{BBs}$ in a higher layer establish call-back procedures at lower-layer BBs. This guarantees that on the syntactic level no mutual relation exists. Design rules exist that avoid that on the semantic level.

BBs are classified into generic and specific BBs. Generic BBs implement the generic part of some functionality. Specific BBs implement the delta part 
of that functionality. The classification in generic and specific functionality is a relative one. A BB is generic or specific with respect to a specific functionality. A BB can be generic and specific with respect to several functionalities. Examples of infrastructure functionality are a device driver abstraction vs. specific device drivers, a BB implementing a fault handling concept vs. those implementing specific faults, etc. One generic $B B$ has usually several specific BBs. A generic BB is always located in a lower layer than a specific $\mathrm{BB}$, i.e., a specific $\mathrm{BBs}$ uses functionality of the generic BB.

Several layers of BBs can be grouped into subsystems [5]. Subsystems follow the rules for layers: subsystems are stacked above each other. To allow configurability even in lower subsystems some of the BBs have only "requires" interfaces, i.e., their functionality is only accessed via call-back interfaces. They can be added and removed without syntactical consequences for BBs in higher subsystems. Subsystem access generics connect functionality from higher subsystems with those configurable BBs.

\section{SOFTWARE ASPECTS}

Software aspects are global functions that cross-cut domain objects. To identify aspects we have to look at a functional structuring of the application domain. Relating these functional structures to the identified objects can lead to 3 cases:

1. a function has relations to, and/or defines functionality of, a few objects only,

2. a function is to be used by almost all objects,

3. a function defines the functionality of almost all objects.

In the first case the function will be handled as (part of) some functional object of the object dimension. In the second case the function will also be handled as a functional object of the object dimension but will be part of the system infrastructure. In the third case the function will be an aspect. Such a function cross-cuts objects. Aspects are a non-hierarchical, potentially complete, functional decomposition of software functionality. The list of aspects should be anchored in the application domain and be defined for a complete product family.

The decision to model such a function as aspect or not depends on the required functionality. Let us take the function of access control as an example. If access control is to be done whenever a user wants to enter a system and, if access is granted, the user is free to use all functionality, access control can be localised as an "access control object" that implements all required functionality. On the other hand, if access control should be more sophisticated depending on users and user groups that have certain 
rights at certain times, the functionality may logically belong to the application objects. A design may use access control lists and a state model for each object to decide if access is granted. Access control could be defined as an aspect of all objects. An implementation could be split into a generic access control object that implements all common functionality, while any other object has to implement its specific access control functionality. The generic component would be part of the system infrastructure. This example shows how the second and third case of function object relations can sometimes be related. A file system is a simple case of such a system where access rights are located with the files while the processing of the access rights is handled in the "file handling object."

To identify aspects we have to look at the functionality from a system perspective. We give several examples of ways to look at system functionality. Some are general, while others stem from specific domains. From these views we derive system aspects. In a further step, ways to derive software aspects from system aspects are described. The identification of aspects has to be done for a specific domain, as it is with objects.

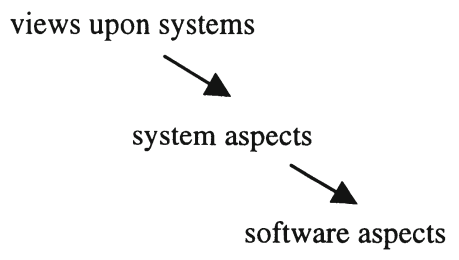

Figure 2. Deriving software aspects

\subsection{Aspects of system design}

System design is a multi-stakeholder and multi-disciplinary task. Besides the functional requirements of the system, non-functional requirements of the customer and the developing organisation have to be met. These different views of a system's functionality constitute the concerns of system design. An architect has to take all these concerns into consideration.

In the following, four different sets of views of a system are presented. They are from different contexts and we do not discuss their pros and cons; they are used as a starting point for the definition of software aspects. In a first step, however, we classify these views according to whether they are directly relevant for system implementation or whether they only shape the context of system implementation. We call views that directly influence system implementation system aspects (figure 2). 


\subsubsection{Functional and non-functional requirements}

Requirements are often classified into functional and non-functional requirements. The intention of this classification is to emphasise the fact that besides functional characteristics many more qualities are expected from a well-designed system. Depending on the system and also the customers, system attributes such as performance, safety, technology choices, testability, reuse, portability, use of standards, etc., are part of the customer requirements or not. A customer can specify these requirements either not at all, partially, or fully. Implicit system attributes that are expected to be present in all systems of a certain class in a specific market segment have to be added. A development organisation will add requirements because of internal benefits or consistency. Therefore the classification into functional and non-functional requirements is, in general, somewhat vague. It cannot directly be used to guide a design. System aspects cover functional and nonfunctional behaviour.

\subsubsection{System quality attributes}

Quality attributes are another view upon the system. Bass et al. [1] classify system qualities in 4 classes:

1. business qualities, such as time to market, cost, projected lifetime of the system, targeted market, roll-out schedule, extensive use of legacy systems

2. quality attributes discernible at run time, such as performance, security, availability, functionality, usability

3. quality attributes not discernible at runtime, such as modifiability, portability, reusability, integrability, testability

4. intrinsic architecture qualities, such as conceptual integrity, correctness and completeness, buildability

These qualities are intended to guide the process of architecting a system. Business qualities determine the context of system implementation. Quality attributes discernible at runtime and those not discernible at runtime are system aspects. Intrinsic architecture qualities guide the process of making an architecture but do not directly influence system implementation.

\subsubsection{Architectural concerns}

G. Muller made a list of architectural concerns [14] for the design of medical imaging systems. He made the point that the system architect has to take all these concerns into consideration (i.e., know the specific 
requirements, communicate with the respective stakeholders, judge on the relative importance, etc.). The architectural concerns are:

- application requirements *,

- functional behaviour *,

- typical load *,

- resource usage (CPU, memory, disk, network, etc.)*,

- installation, configuration, customisation, etc. *,

- factory and field testability *,

- configuration management (technical and commercial) *,

- safety, hazard analysis *,

- security *,

- image quality *,

- functional chain specifications (print, store, etc.)*,

- interoperability, other systems, selected partners, other vendors *,

- interfacing to other applications *,

- technology choices (software, hardware, computer, dedicated digital, make/buy),

- selection and use of mechanisms,

- module design, process design, function allocation (method, file, component, package)*,

- information model: world standardisation, PMS standardisation, PMG standardisation, application specific *,

- test strategy, harnesses, suites, regression,

- verification *,

- performance, throughput, response *,

- re-use consequences, provisions; development process impact; organisational impact; business impact,

- assessment of strong and weak aspects, road map for all views,

- system engineering (cables, cabinets, environment, etc.),

- cost structure (material, production, initial, maintenance, installation),

- logistics, purchasing (long lead items, vulnerability, second sourcing).

These architectural concerns are broad. They look at the system (to be built), its development and use environments. A more restricted view is to look only at system aspects. Architectural concerns that are system aspects are denoted with an asterisk.

\subsubsection{Operator oriented system aspects}

In the area of telecommunication infrastructure, systems tasks and procedures of operators have been classified. A system has to provide interfaces and functionality to support an operator in his or her tasks. A traditional classification distinguishes operation, maintenance, and 
administration tasks; it is often abbreviated OMA. FCAPS is the classification of the OSI system management functional areas (SMFAs) [8]. The functions are divided into fault management, configuration management, accounting management, performance management, and security management. These operator oriented function classifications are system aspects.

\subsection{Mapping of system aspects to software}

Software aspects are derived from system aspects. To do this we first classify system aspects in those that directly specify functionality and those that put constraints on how functionality is implemented. Operator oriented system aspects specify functionality, while system aspects from the system qualities constrain the implementation of functionality. System aspects from the architectural concerns list fall in both classes. Some of them specify functionality that is realised in software. The relation between system aspects and software aspects can be described by the following mappings:

- not relevant for software (e.g., handled in hardware)

- mapped to functional unit (e.g., domain object, BB, subsystem)

- mapped to own software aspect

- mapped to shared software aspect

- distributed over several other software aspects and/or functional blocks

In the following, three examples are given to describe this process. As a first example the aspect list of the tss system where the BBM has been applied first is given. The second example describes the rationale of mapping a system aspect. The third example gives a list of software aspects derived from the architectural concerns list.

\subsubsection{Example: tss software aspects}

As an example we give the list of software aspects in the tss system [2].

- system management

The aspect system management deals with the external control of the system. This may be a man-machine interface, including formatted input and output, or a message-based coded interface.

- recovery

The aspect recovery deals with the proper initialisation of the system during recovery time.

- configuration control

The aspect configuration control deals with the impact of changes in the physical (hardware) and/or logical configuration; changes may have been induced by failures or reconfigurations via system management. 
- data replication

The aspect data replication deals with the replication of data across processor boundaries. Configuration data of the controller are replicated whenever a peripheral device requires a local copy of part of the configuration data.

- test handling

The aspect test handling comprises built-in functions running periodically, or being invoked on specific events, in order to detect and identify internal or external hardware faults or corrupted data. Test functions have no resulting event except to indicate a failure.

- error handling

The aspect error handling is entered when a failure occurs. The related functions take the appropriate actions on a failure. This especially includes damage confinement and fault localisation.

- diagnostics

Functions of the aspect diagnostics are invoked by

- test handling in order to detect faults in the sense of preventive maintenance,

- error handling in order to localise hardware faults,

- configuration control in order to verify the repair or re-configuration of physical or logical objects.

- performance observation

The aspect performance observation deals with the collection and processing of data for statistical and quality measurement purposes.

- debugging

The aspect debugging covers the functions required to debug the online software in test-floor operation as well as filed operation.

- overload control

The aspect overload control implements the functionality to prevent the system from being overloaded. During the overload situation the system is still within the margins of the specified quality of service.

- operational

The aspect operational has a specific character. It represents the core functional behaviour of the system, i.e., handling of calls.

\subsubsection{Example mapping of system aspect reliability in tss}

To illustrate a mapping of a more complex system aspect the implementation of the system aspect reliability in the tss switching system is given.

Reliability is realised in the following ways:

- not in software: 
- the central processor is in a 1:1 redundant configuration that operates in cold stand-by mode

- for the 3 classes of peripheral cards the following holds:

- specific service cards are configured in load sharing or hot standby for dynamically allocated resources

- the network of switching systems implements hot stand by of trunk cards

- subscriber cards are not redundant

- software aspect man-machine interface: changes to the card configuration, states of card and logical objects and parameters thereof made by the operator have transaction semantics

- software aspect configuration: persistence of the configuration is realised in a database

- software aspect error handling: fault management concepts are implemented for card faults to hold the system in a consistent state

\subsubsection{Example of a hypothetical system}

As another example we present a possible definition of software aspects for a hypothetical medical imaging system. The definition is based on the list of system aspects given by G. Muller (see above). A system could have the following list of aspects:

- operational (functionality to process medical images)

- initialisation and recovery

- pixel data communication

- control communication

- imaging information model

- configuration management

- safety and consistency checking

- user interfacing

- testing

These aspects represent functionality that is orthogonal to object modelling. Another concern such as logistics may lead to the use of an identification number for BBs. Comparable cases can be derived from other concerns as well.

\section{ASPECTS AND BUILDING BLOCKS}

Most of the systems designed with the BBM have their features in the object dimension. That means that most of the BBs contain one or more objects completely. Therefore for each $\mathrm{BB}$ a standard substructuring has 
been defined: all aspects are present in each $\mathrm{BB}$, even if some of the aspects are empty for a BB (figure 3). Note that some of the aspects such as debugging may require functionality to be present everywhere. This could mean that a $\mathrm{BB}$ automatically has to implement that functionality. Functionality for another aspect such as error handling is only present in parts of the system where errors can occur (virtual fault absence on higher layers).

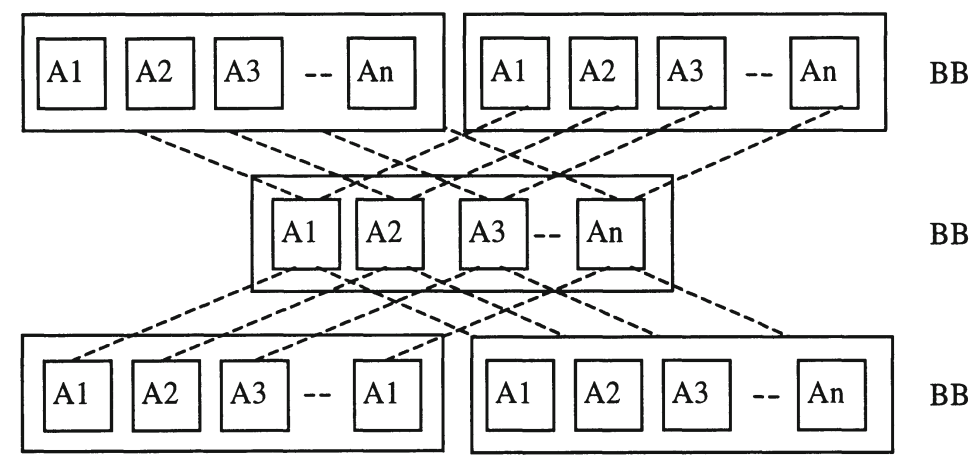

Figure 3. Aspect structuring of building blocks

That means that systems designed with the BBM have BBs as their primary decomposition, with secondary structuring provided by the aspects within the BBs. Introducing new aspects for a system is not forbidden, but it is a worst-case situation where the change effort is almost maximal.

\section{ARCHITECTURAL DESIGN WITH ASPECTS}

The list of aspects is a tool for the architects to check the functional completeness of their identified components. Questions such as: which initialisation actions are required by a component, which faults can a component have, how can it be influenced by other faults in the system, how can the component be configured, what is the required reliability, which resources may it use, etc., help to specify its required functionality.

\subsection{Aspect-completeness of configurable components}

Application features are modelled ideally if they can be added to an installed system. Even more advanced is if the complete system consists of pluggable components. To be able to implement systems from components only, these components have to be functionally complete. Functional 
completeness is relative to a given functional infrastructure. Aspects together with a well-designed infrastructure are a means to achieve this functional completeness. A (set of) $\mathrm{BB}(\mathrm{s})$ is aspect-complete if it allocates all its required resources itself and implements all aspect functionality [15].

\subsection{Aspects and documentation}

In the BB method the notion of a BB is pervasive from architectural design to implementation. In the architectural design $\mathrm{BBs}$ are identified, the specification phase completes the specification of a BB's functionality, in the design and implementation phases it is designed and implemented, respectively. The list of aspects is used for completeness checking in review sessions. Each BB has its own documents: here, again, aspects are used as the main chapters of the document.

\subsection{Aspects and implementation}

In the implementation each function is a triple <object, process, aspect> in the design space, i.e., each function is part of an object, is driven by a process, and is part of an aspect. Making aspects a standard substructuring of a BB, provides a secondary modularity. Naming conventions, files, or programming language modules are possible ways of implementing this modularity. Some of the aspects of a BB may be empty.

\section{COMPARABLE APPROACHES}

The architectural models of Kruchten [11] and Soni et al. [17] also make the distinction between object and process dimensions for their implementation structuring. Kruchten uses the terms development view and process view, while Soni et al. use module interconnection architecture and execution architecture. The examples given by Soni et al. indicate that the conceptual architecture provides a functional decomposition that is hierarchical to the development and the execution architecture. Kruchten's logical view provides no constraints for further structuring in the development and process views.

Kruchten's model is object-oriented and recognises the independence of the modelling of processing resources. It leaves out the aspect dimension, i.e., functions are subordinate to objects. Soni et al. work with a functional structuring and on the next level distinguish between development units and processes. The functional structuring is dominant; object-oriented structuring 
may be used on a micro-level. Perhaps this is the case because their model is more a reverse-architecting model than a forward-architecting model.

The logical model of the BBM is close to the one developed by Kruchten [11], however we can also imagine more function-oriented logical models. The original project where the tss system was developed and from which the BBM originates took only a list of features as the logical model. This may be too limited for domains where most of the domain knowledge is not implicitly present. On the other hand the logical model of Kruchten does not support nor hinder feature-list-like descriptions.

Independent of architectural discussions, limitations of the objectoriented design have been recognised. Kiczales et al. [10] describe examples where object-oriented modelling is too limited and leads to very complex code. He is looking for an alternative structuring that leads to a natural design structure also for more complex examples. Kiczales calls his approach aspect-oriented programming (AOP). He defines an aspect to be functionality that cross-cuts objects. Since his concern is programming and development of next generation programming languages, it could be said that he does bottom-up what the BBM method does top-down, from the system point of view. Furthermore, the need to define (sub-)languages in AOP for each kind of problem creates very specific solutions only.

\section{ACKNOWLEDGEMENTS}

Thanks to Lothar Baumbauer, who, as chief architect, promoted the consequent use of aspects in the tss development. Thanks also to my colleagues Frank van der Linden, Hans Jonkers, Henk Obbink, René Krikhaar, Angelo Hulshout, Jan Gerben Wijnstra and William van der Sterren for valuable discussions and comments to earlier versions of the paper.

\section{REFERENCES}

[1] Len Bass, Paul Clements, Rick Kazman: Software Architecture in Practise, AddisonWesley, 1998

[2] Lothar Baumbauer: System Level Documentation, Volume 6014 (internal documentation) Philips Kommunikations Industrie AG, 1995

[3] Frank Buschmann, Regine Meunier, Hans Rohnert, Peter Sommerlad, Michael Stal: Pattern-oriented Software Architecture - A System of Patterns, Wiley and Sons Ltd., 1996

[4] Paul C. Clements: From Domain Models to Architectures, Workshop on Software Architecture, USC Center for Software Engineering, Los Angeles, 1994

[5] Paul C. Clements: From Subroutines to Subsystems: Component-Based Software

Development, The American Programmer, vol. 8, no. 11, November 1995 
[6] Martin Fowler: UML Distilled, Applying the Standard Object Modelling Language, Addison-Wesley, 1997

[7] Hassan Gomaa: Software Design Methods for Concurrent and Real-Time Systems, Addison-Wesley, 1993

[8] ITU: Management Framework for Open Systems Interconnection (OSI) for CCITT Applications, Recommendation X.700, September 1992

[9] Michael Jackson: Formal Methods and Traditional Engineering, Journal on Systems and Software, vol. 40, pp. 191-194, 1998

[10] Gregor Kiczales, John Lamping, Anurag Mendhekar, Chris Maeda, Cristina Lopes, JeanMarc Loingtier, John Irwing: Aspect-Oriented Programming, Xerox Corporation, 1997

[11] Philipe Kruchten: The 4+1 View Model of Architecture, IEEE Software, Nov.1995

[12] Frank van der Linden and Jürgen K. Müller: Creating Architectures with Building Blocks, IEEE Software, Nov. 1995

[13] Frank van der Linden, Jürgen K. Müller: Composing Product Families from Reusable Components, Bonnie Melhart, Jerzy Rozenblit (eds.) Proceedings 1995 International Symposium and Workshop on Systems Engineering of Computer Based Systems, IEEE, pp. 35 Đ 40 (1995)

[14] Gerrit Muller: Systeem ontwerper een twintig koppig monster?, personal communication

[15] Jürgen K. Müller: Feature-Oriented Software Structuring, CompSAC'97, pp. 552-555, August 1997

[16] Muthu Ramachandran, Wolfgang Fleischer: Design for Large Scale Software Reuse: An Industrial Case Study, 4th Inter'l Conf. on Software Reuse, Orlando, Florida, April 1996

[17] Dilip Soni, Robert L. Nord, and Christine Hofmeister: Software Architecture in Industrial Applications, ICSE'95, Seattle 1995

[18] Clemens Szyperski: Component Software - Beyond Object-Oriented Programming, Essex 1998 